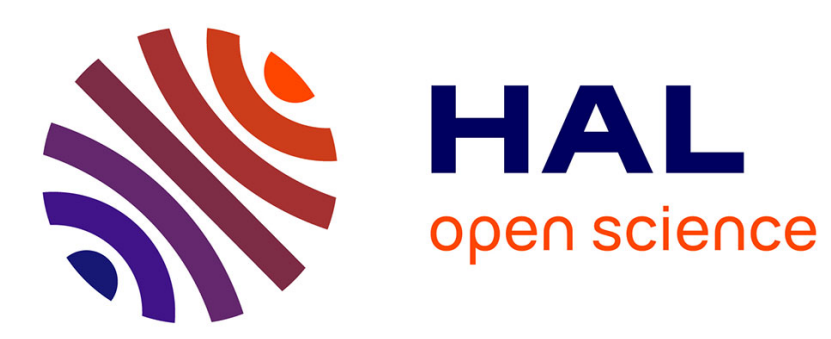

\title{
Palmitoylation of the TRAIL receptor DR4 confers an efficient TRAIL-induced cell death signalling
} Aurélie Rossin, Mathieu Derouet, Fadi Abdel-Sater, Anne-Odile Hueber

\section{To cite this version:}

Aurélie Rossin, Mathieu Derouet, Fadi Abdel-Sater, Anne-Odile Hueber. Palmitoylation of the TRAIL receptor DR4 confers an efficient TRAIL-induced cell death signalling. Biochemical Journal, 2009, 419 (1), pp.185-192. 10.1042/BJ20081212 . hal-00479047

\section{HAL Id: hal-00479047 https://hal.science/hal-00479047}

Submitted on 30 Apr 2010

HAL is a multi-disciplinary open access archive for the deposit and dissemination of scientific research documents, whether they are published or not. The documents may come from teaching and research institutions in France or abroad, or from public or private research centers.
L'archive ouverte pluridisciplinaire HAL, est destinée au dépôt et à la diffusion de documents scientifiques de niveau recherche, publiés ou non, émanant des établissements d'enseignement et de recherche français ou étrangers, des laboratoires publics ou privés. 


\title{
Palmitoylation of the TRAIL receptor DR4 confers an efficient TRAIL-induced cell death signalling
}

\author{
Aurélie Rossin ${ }^{1}$, Mathieu Derouet ${ }^{1}$, Fadi Abdel-Sater ${ }^{1,2,}$, and Anne-Odile Hueber ${ }^{1, *}$ \\ ${ }^{1}$ Equipe labelisée La Ligue; Institute of Developmental Biology and Cancer Research, CNRS \\ UMR 6543, Nice, France. \\ ${ }^{2}$ Current address : Laboratoire de Bioinformatique des Génomes et des Réseaux, Université \\ Libre de Bruxelles, Bruxelles, Belgium; Physiologie Moléculaire de la cellule; Institut de \\ biologie et de Médecine Moléculaires Belgique, Belgium.
}

* Corresponding author: Institute of Signaling, Developmental Biology and Cancer Research, CNRS UMR 6543, Centre A. Lacassagne, 33, Avenue de Valombrose, 06189 Nice, France Tel : 0033 492031241; Fax : 0033 492031245; hueber@unice.fr 


\begin{abstract}
S-palmitoylation is a lipid modification which regulates membrane-protein association and influences protein trafficking, stability or aggregation, thus playing an important role in protein signalling. We previously demonstrated that the palmitoylation of Fas, one of the death-domain (DD)-containing members of the tumor necrosis factor receptors (TNFR) super family, is essential for the redistribution of this receptor into lipid rafts, an obligatory step for the death signal transmission. Here we investigate the requirement of protein palmitoylation in the activities of other DD-containing death receptors. We show that whereas DR4 is palmitoylated, DR5 and TNFR1 are not. Furthermore, DR4 palmitoylation is required for its raft localization and its ability to oligomerize, two essential features in TRAIL induced-death signal transmission.
\end{abstract}

Short title: DR4 palmitoylation and cell death

Keywords: TNFR / signal transduction / cell death / palmitoylation / rafts 


\section{Introduction}

Cell death can be induced by a family of death receptors including Fas (TNFRSF6/CD95/Apo-1), TNFR1 (tumor necrosis factor receptor 1, TNFRSF1A), and the two TNF-related apoptosis-inducing ligand (TRAIL) receptors DR4 (TRAILR1/TNFRSF10A) and DR5 (TRAIL-R2/TNFRSF10B) [1, 2]. Upon activation by their specific ligands, Fas, DR4 and DR5 recruit the adaptor protein FADD (Fas-associated death domain) and caspase 8 through their cytoplasmic death domain (DD) to form the DISC (death-inducing signalling complex), in which the apoptotic cascade is initiated [3-5] . All these receptors are also able to activate non-apoptotic signalling pathways [6-8].

Although the molecular events downstream of DISC formation have been intensively investigated, the initial steps engaging the death receptors to the apoptotic cell fate are more elusive. In this respect, the membrane-proximal events concerning Fas engagement are better understood and a precise sequence of events has been described for Fas-mediated cell death. Several studies have highlighted the constitutive or Fas ligand (FasL)-induced raft microdomain localization of Fas [9-13]. We recently demonstrated that the S-palmitoylation of Fas in its cytoplasmic domain, which consists of the reversible addition of a palmitic acid to a cysteine, represents an essential signal targeting Fas to these rafts [14]. Moreover, palmitoylation allows Fas to associate with the cytoskeletal protein ezrin, reported to be critical for Fas-mediated cell death [15]. Indeed, the FasL-induced internalization of Fas receptor has been shown to occur in the clathrin-dependent pathway $[14,16,17]$ and is a prerequisite for DISC formation, which occurs predominantly in endosomes [16].

However, the mechanisms regulating the initial steps of DR4- or DR5-mediated cell death are poorly understood. Several studies have described a constitutive or a raft relocalisation of DR4 and DR5 upon TRAIL engagement $[18,19]$, as well as receptor-mediated endocytosis through clathrin-dependant and -independent pathways. Nevertheless, this internalization does not seem required for DISC formation and propagation of the death signal [20].

Here, we have investigated the putative role of palmitoylation for TNFR1 and TRAIL receptors. We demonstrate that DR4 is also palmitoylated, and that this post translational modification is critical for DR4 localization in rafts and oligomerisation, two crucial steps in TRAIL-mediated cell death [18, 21$]$.

\section{Results and discussion}

\section{DR4 is palmitoylated but DR5 and TNFR1 are not.}

Protein palmitoylation occurs on cysteine residues located in or near the transmembrane domain. We and others have previously demonstrated that human Fas is constitutively palmitoylated on cysteine 199 [14, 22]. Sequence analysis revealed that DR4, DR5 and TNFR1 also possess one or more cysteines that are potential palmitoylation sites (Fig. 1A in blue). The palmitoylation status of the receptors was assessed by incubating HEK293 cells transiently expressing Fas, DR4, DR5 or TNFR1 with $\left[{ }^{3} \mathrm{H}\right]$ palmitate. Immunoprecipitation of the receptors followed by immunoblotting and autoradiography showed an incorporation of $\left[{ }^{3} \mathrm{H}\right]$ palmitate by DR4 (Fig. 1B), which could be abolished by competition with the two palmitate analogues 2-bromo-palmitate (BrP, Fig. 1B) and 13-oxypalmitate (13-OP, Fig. 1B) [23], demonstrating that DR4 is palmitoylated. Under these conditions, we did not detect any palmitoylation of DR5 or TNFR1, suggesting that palmitoylation is not a general post translational modification for DD receptors. The results of this metabolic $\left[{ }^{3} \mathrm{H}\right]$ palmitate labelling were further confirmed by using a non-radioactive method relying on an acyl- 
biotinyl exchange chemistry, in which the S-palmitoyl thioesther link is specifically cleaved by an hydroxylamine exposure and substituted by a biotin moiety (Supplementary Fig.1A).

Using site directed mutagenesis, we mutated the four putative palmitoylation sites present in DR4 and obtained four DR4 mutants: DR4 C261-3S, in which the cysteine triplet was mutated, DR4 C268S, DR4 C274S and DR4 C279S. Plasmids encoding DR4 WT and the four mutants were transfected into HEK293 cells and the palmitoylation of the receptors was assessed. A lack of $\left[{ }^{3} \mathrm{H}\right]$ palmitate incorporation was observed only when cysteines 261 to 263 were mutated. Thus, DR4 palmitoylation occurs within this cysteine triplet (Fig. 1C). In order to further identify palmitoylated cysteine residue, we generated the single mutants, C261S, C262S, and C263S. However, none of the single mutations could abolish DR4 palmitoylation (Supplementary Fig.1B), thus rendering the identification of a unique amino acid as the palmitoylation site impossible. This result might reflect at least two possible situations that might be even combined: (i) several cysteines are palmitoylated at once; (ii) only one cysteine is palmitoylated but the palmitoylation site can be interchangeable probably due to the close proximity of the two other cysteine residue.

\section{DR4 palmitoylation is required for TRAIL-mediated cell death.}

The function of DR4 palmitoylation was first investigated using a pharmacologic approach. The Human Embryonic Kidney cells HEK293, which express DR4 and DR5 at a basal level were transiently transfected with DR4 or DR5 to predominantly express DR4 or DR5 respectively. Pre-treatment with the palmitate analogue 13-oxypalmitate (13-OP) [23] induced $60 \%$ inhibition of cell death after TRAIL engagement on DR4 transfected cells demonstrating the important role of palmitoylation in TRAIL-mediated cell death (Fig. 2A). Interestingly, TRAIL-induced cell death was not affected by the 13-OP preincubation on DR5 overexpressing cells, indicating that the 13-OP inhibitory action is restricted to DR4-mediated signalling (Fig.2A). These results were confirmed at the molecular level on PARP cleavage (Fig.2B).

We then analyzed the function of endogenous DR4 palmitoylation in three different cell lines which express both DR4 and DR5: the HEK293 cell line, the chronic myeloid leukaemia cell line AR230 and the breast cancer cell line MDA-MB-231. The DR4 endogenous palmitoylation was checked using the acyl-biotin exchange technique (Supplementary Fig.1C). We generated cells expressing DR4 as the main DD containing TRAIL receptor by silencing DR5 in the three cell lines using lentivirus expressing shRNA. (Fig.2C). As expected, the DR5 knockdown cells were less sensitive to TRAIL-induced cell death compared to cells expressing the scramble sequence (Fig. 2C). A two-hour pre-incubation with 13-OP induced between 40 and $50 \%$ inhibition of TRAIL-induced cell death in the three cell lines expressing the scramble shRNA, indicating a major pro-apoptotic role for palmitoylation in TRALL-mediated cell death. Interestingly, the same level of cell death inhibition was achieved by 13-OP pre-treatment when the DR5 expression was silenced, suggesting a specific contribution of DR4 palmitoylation in TRAIL-induced death signalling. A PARP cleavage analysis confirmed the results obtained with cell death (Fig.2D).

To investigate the specific role of DR4 palmitoylation with a genetic approach, we transfected HEK293 shDR5 cells with an empty vector, DR4 WT and DR4 C261-3S. After verification of equal DR4 expression at the cell surface (data not shown), cell death was monitored following TRAIL stimulation (Fig. 3A). Inclusion of the empty vector in the experiment allowed the role of endogenous DR4 to be distinguished. Upon TRAIL engagement, a significant induction of cell death was observed and could be clearly attributed to the transfected DR4 after 5 hours of TRAIL treatment (compare pcR3- and pcR3 DR4 WT-transfected cells). Importantly, cell death of HEK293 shDR5 cells transfected with pcR3 DR4 C261-3S was significantly reduced compared to DR4 WT transfected cells demonstrating that this palmitoylation-deficient form of DR4 is less efficient to transmit a death signal. One can note 
that the mutant DR4 keeps some ability to induce cell death (compare pcR3- and pcR3 DR4 C261-3S-transfected cells). This decrease in cell death was confirmed at the molecular level as an inhibition of caspase 8 and PARP cleavage (Fig. 3B).

We next investigated whether DISC formation was altered in presence of the palmitoylationdeficient DR4 mutant: a specific recruitment of caspase 8 and FADD to activated DR4 was observed upon TRAIL engagement in the presence of the wild-type receptor. In contrast, this recruitment was abolished when DR4 C261-3S was expressed (Fig. 2E).

Altogether, our results demonstrate that DR4 palmitoylation is crucial for DR4 DISC formation upon TRAIL engagement and transmission of the death signal.

\section{DR4 palmitoylation is critical for raft localization.}

Palmitoylation can be used as a signal targeting proteins to the raft microdomains [14, 24]. We therefore investigated whether DR4 was located in lipid rafts. Post-nuclear supernatant (PNS) from HEK293, AR230 and MDA-MB-231 cells were solubilised with polyethylene ether Brij 98 and subjected to ultracentrifugation through a sucrose density gradient. The detergent-resistant microdomains (DRM) rafts were found in the low-density fractions (LF). Sucrose fractions were tested by immunoblotting, using caveolin and clathrin heavy chain (CHC) as raft and non-raft markers respectively. Interestingly, both DR4 and DR5 were found almost exclusively in the rafts in all tested cell lines (Fig. 4A). No change in raft localization was observed for DR4 upon TRAIL stimulation (data not shown) nor when DR5 expression was silenced (supplementary Fig.2). Conflicting data have been obtained concerning DR4 and DR5 raft localization in several studies. Our results are in agreement with those obtained by Song et al who observed constitutive raft localisation for both receptors in non-small cell lung carcinoma [18]. However, this is in contradiction with others studies describing constitutive non-raft localisation for both DR4 and DR5 and raft recruitment only upon TRAIL stimulation [19] or drug treatment [25]. These discrepancies could be attributed to the use of different cell lines and different raft isolation techniques [26].

To address the question of the functional significance of DR4 and DR5 raft localization, we depleted the cells of cholesterol using cholesterol oxidase treatment, which disorganizes the lipid rafts. Using conditions that induce a partial loss of death receptor raft localisation (Fig. 4B, we observed a $60 \%$ inhibition of TRAIL-mediated cell death in both HEK293 cells overexpressing DR4 and AR230 cells following pre-treatment with cholesterol oxidase (Fig. 4B). In contrast, staurosporine-induced apoptosis was not affected by cholesterol oxidase treatment, confirming the specificity of the treatment to TRAIL signalling (data not shown). Thus, DR4 and DR5 raft localisation are necessary for TRAIL-mediated cell death. Similar conclusions were driven by Song et al using another cholesterol depleting agent methyl- $\beta$ cyclodextin [18].

To directly evaluate the contribution of DR4 palmitoylation to DR4 raft localisation, HEK293 cells were subjected to lipid raft preparation with or without 13-OP treatment. 13-OP exposure induced a 50\% delocalisation of endogenous DR4 from the lipid raft whereas DR5 localisation remains unchanged, suggesting that palmitoylation represents a major signal targeting DR4 to the lipid rafts. These results were confirmed by a genetic approach using HEK293 cells transfected with DR4 WT and DR4 C261-3S. Unlike the WT receptor, the majority of the palmitoylation-deficient DR4 was found in the non-raft fraction, clearly demonstrating that DR4 palmitoylation is necessary for raft localisation (Fig. 4D). However, an alternative signal targeting DR4 to the rafts appears to exist, since a small proportion of the DR4 C261-3S could still be found in the raft fraction. Interestingly, DR5 (Fig. 4A) and TNFR1 (data not shown) are also directed to the rafts by a palmitoylation-independent mechanism since these two receptors are associated to lipid rafts despite the fact that they are not palmitoylated (Fig. 1B). For TNFR1, the death domain itself was reported to be necessary and sufficient for this localization [27]. 


\section{Palmitoylation influences DR4 oligomerization.}

Using non-reducing conditions for raft sample preparation, we observed that, in addition to causing relocalisation of the monomer form of DR4 into non-raft fractions (Fig. 4D), the mutation also affected higher oligomer forms (data not shown). Immunoblotting of nonreducing total lysates with DR4 antibody showed very different migration patterns for DR4 WT and DR4 C261-3S. Three different oligomers bands were observed for the WT receptor (Fig.5A), while only one band ( $\left.\mathrm{n}^{\circ} 1\right)$ was predominant in the C261-3S DR4 sample. Bands 2 and 3 were only visible after long exposure of the film. Thus, not only the non-raft localisation, but also this altered oligomerization capacity of the palmitoylation mutant could explain its low ability to induce cell death.

Moreover, while DR4 WT presented a doublet band for the monomer, only the lower one was observed for the palmitoylation-deficient mutant, suggesting that the upper band represents palmitoylated DR4, which can be detected in the absence of reducing agents. Thus, only some of DR4 WT is palmitoylated.

DR4 is able to form homo oligomers comprising several DR4 molecules, but also hetero oligomers with other TRAIL receptors such as DR5 $[4,19]$. In order to investigate the composition of oligomers formed by DR4 WT and DR4 C261-3S, we performed coimmunoprecipitations with anti-DR4 or anti-DR5 antibodies of HEK293 cells transfected with the indicated plasmids (Fig. 4B) and immunoblotted them using anti-DR4 antibody. While the oligomerization pattern obtained after the DR 4 immunoprecipitation was similar to the one obtained from total lysates, only band $n^{\circ} 2$ contained DR5 and no DR4 C261-3S/DR5 interaction was observed.

All together our results pointed out the essential role of the post-translational modification of the death receptor DR4 by palmitoylation which we show required for the lipid rafts localization of the receptor, and therefore for an efficient transmission of the cell death signal. Interestingly we reported that DR4 palmitoylation is not only required for its localization to lipid rafts but also for its homo-oligomerization and for its association with DR5 (hetero-oligomerization). Future studies will be needed to investigate the exact functional correlation of lipid raft association of the TRAIL receptors, the formation of the DR4-DR4 or DR4-DR5 complexes and their death-inducing capacities. 


\section{References.}

1 Ashkenazi, A. and Dixit, V. M. (1998) Death receptors: signaling and modulation. Science 281, 1305-1308

2 Peter, M. E., Scaffidi, C., Medema, J. P., Kischkel, F. and Krammer, P. H. (1999) The death receptors. Results Probl Cell Differ 23, 25-63

3 Kischkel, F. C., Hellbardt, S., Behrmann, I., Germer, M., Pawlita, M., Krammer, P. H. and Peter, M. E. (1995) Cytotoxicity-dependent APO-1 (Fas/CD95)-associated proteins form a death-inducing signaling complex (DISC) with the receptor. Embo J 14, 5579-5588

4 Kischkel, F. C., Lawrence, D. A., Chuntharapai, A., Schow, P., Kim, K. J. and Ashkenazi, A. (2000) Apo2L/TRAIL-dependent recruitment of endogenous FADD and caspase-8 to death receptors 4 and 5. Immunity 12, 611-620

5 Sprick, M. R., Weigand, M. A., Rieser, E., Rauch, C. T., Juo, P., Blenis, J., Krammer, P. H. and Walczak, H. (2000) FADD/MORT1 and caspase-8 are recruited to TRAIL receptors 1 and 2 and are essential for apoptosis mediated by TRAIL receptor 2 . Immunity 12, 599-609

6 Muppidi, J. R., Tschopp, J. and Siegel, R. M. (2004) Life and death decisions: secondary complexes and lipid rafts in TNF receptor family signal transduction. Immunity 21, 461-465

7 Peter, M. E., Budd, R. C., Desbarats, J., Hedrick, S. M., Hueber, A. O., Newell, M. K., Owen, L. B., Pope, R. M., Tschopp, J., Wajant, H., Wallach, D., Wiltrout, R. H., Zornig, M. and Lynch, D. H. (2007) The CD95 receptor: apoptosis revisited. Cell 129, 447-450

8 Falschlehner, C., Emmerich, C. H., Gerlach, B. and Walczak, H. (2007) TRAIL signalling: decisions between life and death. Int J Biochem Cell Biol 39, 1462-1475

9 Hueber, A. O., Bernard, A. M., Herincs, Z., Couzinet, A. and He, H. T. (2002) An essential role for membrane rafts in the initiation of Fas/CD95-triggered cell death in mouse thymocytes. EMBO Rep 3, 190-196

10 Henkler, F., Behrle, E., Dennehy, K. M., Wicovsky, A., Peters, N., Warnke, C., Pfizenmaier, K. and Wajant, H. (2005) The extracellular domains of FasL and Fas are sufficient for the formation of supramolecular FasL-Fas clusters of high stability. J Cell Biol 168, 1087-1098

11 Miyaji, M., Jin, Z. X., Yamaoka, S., Amakawa, R., Fukuhara, S., Sato, S. B., Kobayashi, T., Domae, N., Mimori, T., Bloom, E. T., Okazaki, T. and Umehara, H. (2005) Role of membrane sphingomyelin and ceramide in platform formation for Fasmediated apoptosis. J Exp Med 202, 249-259

12 Gajate, C., Del Canto-Janez, E., Acuna, A. U., Amat-Guerri, F., Geijo, E., SantosBeneit, A. M., Veldman, R. J. and Mollinedo, F. (2004) Intracellular triggering of Fas aggregation and recruitment of apoptotic molecules into Fas-enriched rafts in selective tumor cell apoptosis. J Exp Med 200, 353-365

13 Beneteau, M., Pizon, M., Chaigne-Delalande, B., Daburon, S., Moreau, P., De Giorgi, F., Ichas, F., Rebillard, A., Dimanche-Boitrel, M. T., Taupin, J. L., Moreau, J. F. and Legembre, P. (2008) Localization of Fas/CD95 into the Lipid Rafts on DownModulation of the Phosphatidylinositol 3-Kinase Signaling Pathway. Mol Cancer Res 6, 604-613

14 Chakrabandhu, K., Herincs, Z., Huault, S., Dost, B., Peng, L., Conchonaud, F., Marguet, D., He, H. T. and Hueber, A. O. (2007) Palmitoylation is required for efficient Fas cell death signaling. Embo J 26, 209-220

15 Parlato, S., Giammarioli, A. M., Logozzi, M., Lozupone, F., Matarrese, P., Luciani, F., Falchi, M., Malorni, W. and Fais, S. (2000) CD95 (APO-1/Fas) linkage to the actin 
cytoskeleton through ezrin in human T lymphocytes: a novel regulatory mechanism of the CD95 apoptotic pathway. Embo J 19, 5123-5134

16 Lee, K. H., Feig, C., Tchikov, V., Schickel, R., Hallas, C., Schutze, S., Peter, M. E. and Chan, A. C. (2006) The role of receptor internalization in CD95 signaling. Embo J 25, 1009-1023

17 Chakrabandhu, K., Huault, S., Garmy, N., Fantini, J., Stebe, E., Mailfert, S., Marguet, D. and Hueber, A. O. (2008) The extracellular glycosphingolipid-binding motif of Fas defines its internalization route, mode and outcome of signals upon activation by ligand. under revision

18 Song, J. H., Tse, M. C., Bellail, A., Phuphanich, S., Khuri, F., Kneteman, N. M. and Hao, C. (2007) Lipid rafts and nonrafts mediate tumor necrosis factor related apoptosis-inducing ligand induced apoptotic and nonapoptotic signals in non small cell lung carcinoma cells. Cancer Res 67, 6946-6955

19 Merino, D., Lalaoui, N., Morizot, A., Schneider, P., Solary, E. and Micheau, O. (2006) Differential inhibition of TRAIL-mediated DR5-DISC formation by decoy receptors 1 and 2. Mol Cell Biol 26, 7046-7055

20 Kohlhaas, S. L., Craxton, A., Sun, X. M., Pinkoski, M. J. and Cohen, G. M. (2007) Receptor-mediated endocytosis is not required for tumor necrosis factor-related apoptosis-inducing ligand (TRAIL)-induced apoptosis. J Biol Chem 282, 1283112841

21 Wagner, K. W., Punnoose, E. A., Januario, T., Lawrence, D. A., Pitti, R. M., Lancaster, K., Lee, D., von Goetz, M., Yee, S. F., Totpal, K., Huw, L., Katta, V., Cavet, G., Hymowitz, S. G., Amler, L. and Ashkenazi, A. (2007) Death-receptor Oglycosylation controls tumor-cell sensitivity to the proapoptotic ligand Apo2L/TRAIL. Nat Med 13, 1070-1077

22 Feig, C., Tchikov, V., Schutze, S. and Peter, M. E. (2007) Palmitoylation of CD95 facilitates formation of SDS-stable receptor aggregates that initiate apoptosis signaling. Embo J 26, 221-231

23 Hawash, I. Y., Hu, X. E., Adal, A., Cassady, J. M., Geahlen, R. L. and Harrison, M. L. (2002) The oxygen-substituted palmitic acid analogue, 13-oxypalmitic acid, inhibits Lck localization to lipid rafts and T cell signaling. Biochim Biophys Acta 1589, $140-$ 150

24 Resh, M. D. (2006) Palmitoylation of ligands, receptors, and intracellular signaling molecules. Sci STKE 2006, re14

25 Gajate, C. and Mollinedo, F. (2007) Edelfosine and perifosine induce selective apoptosis in multiple myeloma by recruitment of death receptors and downstream signaling molecules into lipid rafts. Blood 109, 711-719

26 Hueber, A. O. (2003) Role of membrane microdomain rafts in TNFR-mediated signal transduction, Cell Death Differ 10, 7-9

27 Cottin, V., Doan, J. E. and Riches, D. W. (2002) Restricted localization of the TNF receptor CD120a to lipid rafts: a novel role for the death domain. J Immunol 168, 4095-4102

28 Herincs, Z., Corset, V., Cahuzac, N., Furne, C., Castellani, V., Hueber, A. O. and Mehlen, P. (2005) DCC association with lipid rafts is required for netrin-1-mediated axon guidance. J Cell Sci 118, 1687-1692

29 Drisdel, R. C. and Green, W. N. (2004) Labeling and quantifying sites of protein palmitoylation. Biotechniques 36, 276-285

30 Wan, J., Roth, A. F., Bailey, A. O. and Davis, N. G. (2007) Palmitoylated proteins: purification and identification. Nat Protoc 2, 1573-1584 


\section{Acknowledgements.}

We thank Sebastien Huault for technical assistance, Zoltan Hérincs for advices on raft preparation and palmitoylation experiments, Olivier Micheau for providing the DR4, DR5 and TNFR1 constructs and advice during the initial steps of the project, Hai-Tao He and Krittalak Chakrabandhu for helpful discussions.

This work was supported by institutional funds from the Centre National de la Recherche Scientifique (CNRS) and by grants from the Ligue nationale contre le cancer (LNCC), INCA (Institut National du Cancer) and ANR (Agence Nationale de la Recherche). AR is supported by a postdoctoral fellowship from the Association pour la Recherche sur le Cancer (ARC). 


\section{Material and methods}

\section{Antibodies and reagents}

Flag-rhTRAIL was obtained from Alexis. The DR4 and DR5 antibodies used for immunoblotting were purchased from Chemicon and Diaclone, and those used for immunoprecipitation were from Alexis. The TNFR1 antibodies used for immunoblotting and immunoprecipitation were from Santa Cruz and R\&D, respectively. Anti-caspase 8 was purchased from Cell Signalling, anti-ezrin from Zymed, anti-FADD from MBL, anti-caspase 3 from BD Bioscience, anti-PARP from Biomol, and anti-Fas (C20), anti-clathrin heavy chain and anti-caveolin-1 from Santa Cruz.

\section{Constructs}

pcR3 DR4 C261-3S, pcR3 DR4 C261S, pcR3 DR4 C262S, pcR3 DR4 C263S, pcR3 DR4 C268S, pcR3 DR4 C274S and pcR3 DR4 C279S were obtained using the Quikchange sitedirected mutagenesis kit (Qiagen) using pcR3 DR4 as a template and the following primers:

GTGGCTGTGCTGATTGTCAGTAGTAGCATCGGCTCAGGTTGTGG (forward C261-3S) CCACAACCTGAGCCGATGCTACTACTGACAATCAGCACAGCCAC (reverse C261-3S) CTGATTGTCAGTTGTTGCATCGGCTCAGG (forward C261S)

CCTGAGCCGATGCAACAACTGACAATCAG (reverse C261S)

CTGATTGTCTGTAGTTGCATCGGCTCAGG (forward C262S)

CCTGAGCCGATGCAACTACAGACAATCAG (reverse C262S)

CTGATTGTCTGTTGTAGCATCGGCTCAGG (forward C263S)

CCTGAGCCGATGCTACAACAGACAATCAG (reverse C263S)

TGCATCGGCTCAGGTAGCGGAGGGGACCCCAAG (forward C268S)

CTTGGGGTCCCCTCCGCTACCTGAGCCGATGCA (reverse C268S)

GAGGGGACCCCAAGAGCATGGACAGGGTGTG (forward C274S)

CACACCCTGTCCATGCTCTTGGGGTCCCCTC (reverse C274S)

GCATGGACAGGGTGAGTTTCTGGCGCTTGGG (forward C279S)

CCCAAGCGCCAGAAACTCACCCTGTCCATGC (reverse C279S)

Lentivirus expressing five different short hairpin RNA (shRNA) targeting DR5 were obtained from Sigma (MISSION ${ }^{\circledR}$ shRNA Lentiviral Transduction Particles). The choosen sequence for the shRNA is CCGGGCAGAAGATTGAGGACCACTTCTCGAGAAGTGGTCCTCAATCTTCTGC TTTTT. A control non-target shRNA lentiviral transduction particle expressing a scramble sequence for shRNA was also used.

\section{Cell culture, transfection, lentiviral infection and and cell lysis}

HEK293 human embryonic kidney cells and MDA-MB-231 human epithelial breast cancer cells were grown in DMEM supplemented with 10\% foetal calf serum. AR230 human chronic myeloid leukaemia cells were grown in RPMI supplemented with $10 \%$ foetal calf serum. HEK293 cells were transiently transfected using the calcium phosphate technique, as previously described [28]. For palmitic acid competition with palmitic acid analogues, cells were pre-incubated with $100 \mu \mathrm{M}$ 2-bromo palmitate (Sigma) or $300 \mu \mathrm{M}$ 13-oxypalmitate for 2 hours in serum-free conditions. For cholesterol depletion, cells were pre-incubated with cholesterol oxidase (Calbiochem) for 2 hours in serum-free medium. HEK293, MDA-MB231 and AR230 cells were lysed for Western blotting analysis with Laemmli buffer. 
For lentiviral infections, HEK293, MDA-MB-231 and AR230 cells were infected with lentiviruses expressing five different shRNA sequences targeting DR5 or one shRNA scramble sequence with a MOI of 1:1. Cells were then selected for stable integration with 1 $\mu \mathrm{g} / \mathrm{ml}$ puromycin, the efficiency of the knock-down was assessed by western blot and one population obtained from one single sequence was chosen. These populations were called HEK293, AR230 or MDA-MB-231 shDR5 and compared to populations infected with the scramble shRNA (shscramble).

\section{Protein palmitoylation detection.}

We first used a metabolic $\left[{ }^{3} \mathrm{H}\right]$ palmitate labelling to detect protein palmitoylation. HEK293 cells transfected with the indicated plasmids were incubated for 5 hours at $37^{\circ} \mathrm{C}$ with $\left[9,10(\mathrm{n})^{3} \mathrm{H}\right]$ palmitic acid (Amersham, specific activity $60 \mathrm{Ci} / \mathrm{mmol}$ ) at $0.2 \mathrm{mCi} / \mathrm{ml}$ in serumfree medium. After washing, cells were lysed in buffer A (25 mM HEPES, $150 \mathrm{mM} \mathrm{NaCl}, 1$ mM EGTA, protease inhibitor cocktail) containing 1\% Nonidet P-40 (NP-40) and 10\% glycerol (lysis buffer) at $4^{\circ} \mathrm{C}$, and immunoprecipitated with the indicated antibodies coupled to Protein A/G plus agarose beads (Tebu) at $4^{\circ} \mathrm{C}$ overnight. Immunoprecipitates were submitted to SDS-PAGE. Gels were fixed, enhanced using Amplify (Amersham) and exposed for autoradiography at $-80^{\circ} \mathrm{C}$ for 10 and 30 days.

An alternative method to detect protein palmitoylation has been used and adapted from the acyl-biotinyl exchange protocol of Drisdel and Green [29, 30] which comprises three chemical steps : (i) cell lysates in 250mM HEPES, $1 \mathrm{mM}$ EDTA and 2,5\% SDS were incubated with $0,1 \%$ methyl methanethiosulfonate (sigma) at $50^{\circ} \mathrm{C}$ for 20 minutes to block free thiols. (ii) Proteins were precipitated with acetone and resuspended in $1 \mathrm{M}$ hydroxylamine pH 7.4 (sigma) which specifically release thioesther-linked palmitoyl moieties and restore the modified cysteines to thiols, (iii) which were then mixed with $0,2 \mathrm{mM}$ biotinHPDP (Pierce) for 1 hour at room temperature. Biotinylated proteins were purified with neutravidin beads (Pierce), separated by SDS-PAGE and submitted to immunoblotting with antibody against the protein of interest. Specificity of the experiment is controlled by omitting the hydroxylamine treatment.

\section{DISC isolation}

HEK293 cells transfected with pcR3 DR4 WT or pcR3 DR4 C261-3S were stimulated as indicated and lysed with buffer A containing 1\% NP-40 and 10\% glycerol (lysis buffer) at 4 ${ }^{\circ} \mathrm{C}$. The PNS obtained after centrifugation at $800 \mathrm{~g}$ for 10 minutes at $4^{\circ} \mathrm{C}$ were incubated with DR4 antibody (Alexis) overnight and immunoprecipitated with Protein G-sepharose beads (Zymed). After 4 washes with lysis buffer, beads were eluted with Laemmli buffer and submitted to SDS-PAGE, followed by immunoblotting.

\section{Raft isolation}

Briefly, PNS from HEK293, AR230, and MDA-MB-231 cells $\left(2 \times 10^{7}\right)$ were solubilised with $1 \%$ Brij 98 for 1 hour at $4{ }^{\circ} \mathrm{C}$ and diluted with $2 \mathrm{ml}$ buffer A containing $2 \mathrm{M}$ sucrose, before being placed at the bottom of a step sucrose gradient (1.33-0.9-0.8-0.75-0.7-0.6-0.5-0.4-0.3$0.2 \mathrm{M}$ ) in buffer A. After 16 hours centrifugation at $4^{\circ} \mathrm{C}$ at $38000 \mathrm{rpm}$ in a SW41 rotor (Beckman Coulter), eight $1 \mathrm{ml}$ fractions and one $3 \mathrm{ml}$ fraction (fraction 9) were harvested from the top.

\section{Cell death assays}

HEK293 cells transfected or not with the indicated plasmids, AR230 cells or MDA-MB-231 cells were incubated with the indicated doses of Flag-rhTRAIL plus $1 \mu \mathrm{g} / \mathrm{ml} \mathrm{M} 2$ for different time periods. HEK293 cell death was measured by morphological observation of trypan blue- 
stained cells. Each sample was counted four independent times per experiment in at least 3 different experiments.

AR230 cells and MDA-MB-231 cells were fixed in ice-cold 70\% ethanol, washed in $38 \mathrm{mM}$ citrate (pH 7.4) and incubated for 20 minutes in $38 \mathrm{mM}$ citrate supplemented with $69 \mu \mathrm{M}$ propidium iodide (Sigma) and $5 \mu \mathrm{g} / \mathrm{ml}$ RNaseA (Sigma). Cells were analysed with a FACSCalibur cytometer (Becton Dickinson) and the proportion of sub-G1 particles was determined. 


\section{Figure legends}

Figure 1: DR4 is palmitoylated in the cytoplasmic region. (A) Sequence comparison of the membrane-proximal cytoplasmic region of human Fas, TNFR1, DR4 and DR5. The transmembrane sequence is represented in red, the cytoplasmic part in black and cysteine residues providing putative sites for palmitoylation are highlighted in blue. (B) DR4 is palmitoylated. HEK293 cells transiently expressing Fas, DR4, DR5 or TNFR1 were subjected to metabolic labelling with $\left[{ }^{3} \mathrm{H}\right]$ palmitate. Death receptors were immunoprecipitated with specific antibody and analysed by both autoradiography (bottom part) and immunoblotting (ECL). The palmitic analogues bromo-palmitate (BrP) and 13-oxypalmitate (13-OP) were used to compete with $\left[{ }^{3} \mathrm{H}\right]$ palmitate and confirm the specificity of the labelling (right panel). (C) DR4 is palmitoylated on the cysteine triplet at 261-3. The four putative palmitoylation sites were mutated from cysteine to serine by site-directed mutagenesis and the palmitoylation status was assessed as in (B).

Figure 2: The palmitic acid analogue 13-OP inhibits TRAIL-induced DR4-mediated cell death. (A) HEK293 cells transiently transfected with pcR3 DR4 or pcR3 DR5 were preincubated with $300 \mu \mathrm{M}$ of the palmitic acid analogue 13-OP for 2 hours or left untreated. Cell death was monitored after 3 hours of $250 \mathrm{ng} / \mathrm{ml}$ Flag-rhTRAIL plus $1 \mu \mathrm{g} / \mathrm{ml}$ M2. (B) HEK293 cells transiently expressing DR4 or DR5 were treated as in (A). Cell lysates were separated by SDS-PAGE and submitted to immunoblotting to assess PARP cleavage. (C) HEK293, AR230 and MDA-MB-231 cells expressing shRNA targeting DR5 or a scramble (sc) sequence were pre-treated for 2 hours with $300 \mu \mathrm{M} 13-\mathrm{OP}$ and then exposed to $250 \mathrm{ng} / \mathrm{ml}$ Flag-rhTRAIL plus $1 \mu \mathrm{g} / \mathrm{ml} \mathrm{M2}$. Cell death was detected after five (HEK293 and AR230) or four hours (MDA-MB-231). Efficient DR5 knock-down was controlled by western blot. (D) MDA-MB231 shDR5 or MDA-MB-231 scramble cells were treated as in (C). Cells were lysed with laemmli buffer and PARP cleavage was assessed by western blot.

Figure 3: DR4 palmitoylation on the cysteine triplet 261-3 is required for efficient TRAIL-induced cell death. (A) HEK293 shDR5 cells transiently transfected with pcR3, pcR3 DR4 WT or pcR3 DR4 C261-3S were subjected to TRAIL-induced cell death with the indicated Flag-rhTRAIL dose plus $1 \mu \mathrm{g} / \mathrm{ml} \mathrm{M2}$. Cell death was detected after 5 hours incubation. (B) HEK293 cells transiently transfected with pcR3 DR4 WT or pcR3 DR4 C261$3 \mathrm{~S}$ and treated with $250 \mathrm{ng} / \mathrm{ml}$ Flag-rhTRAIL plus $1 \mu \mathrm{g} / \mathrm{ml} \mathrm{M} 2$ were lysed after 3 hours treatment and submitted to Western blotting. (C) DISC isolation was performed on HEK293 cells transiently expressing DR4 WT or DR4 C261-3S and stimulated for 15 minutes with 250 ng/ml Flag-rhTRAIL plus $1 \mu \mathrm{g} / \mathrm{ml}$ M2.

Figure 4: Palmitoylation is required to target DR4 to the rafts. (A) Brij 98-solubilised PNS from HEK293, AR230 and MDA-MB-231 cells were submitted to low-density, detergent-resistant membrane microdomain (DRM) preparation. DRMs are localized in fractions 1-4 and referred as light fractions (LF). HF denotes high-density fractions where detergent-solubilized proteins or those associated with high-density microdomains are localized. Caveolin and CHC serve as markers for LF and HF, respectively. The nine resulting sucrose fractions were immunoblotted with the indicated antibodies. (B) HEK293 cells transfected with pcR3 DR4 and AR230 cells were preincubated for 2 hours with $2 \mathrm{U} / \mathrm{ml}$ cholesterol oxidase. Cell death was monitored after 3 hours of $250 \mathrm{ng} / \mathrm{ml}$ Flag-rhTRAIL plus $1 \mu \mathrm{g} / \mathrm{ml} \mathrm{M2.} \mathrm{In} \mathrm{parallel,} \mathrm{DR4} \mathrm{and} \mathrm{DR5} \mathrm{DRM} \mathrm{delocalisation} \mathrm{was} \mathrm{confirmed} \mathrm{by} \mathrm{submitted}$ HEK293 cells treated with the indicated dose of cholesterol oxidase to DRM preparation. (C) HEK293 cells were treated with $300 \mu \mathrm{M} 13-\mathrm{OP}$ for 2 hours before DRM preparation as in 
(A). Endogenous DR4 delocalisation from the raft was monitored by western blot. (D) HEK293 cells transfected with pcR3 DR4 WT or pcR3 DR4 C261-3S were subjected to raft preparation as in (A) and exogenous DR4 expression detected by immunoblotting.

Figure 5: DR4 oligomerization profile is altered in the palmitoylation-deficient mutant. (A) Non-reducing SDS-PAGE of total lysates from HEK293 cells transfected with pcR3 DR4 WT or pcR3 DR4 C261-3S immunoblotted with anti-DR4 antibody. (B) HEK293 cells were transfected with vectors encoding the indicated proteins. Immunoprecipitation with anti-DR4 antibody (left panel) and anti-DR5 antibody (right panel) was followed by SDS-PAGE under non-reducing conditions and immunoblotting with anti-DR4 antibody. 
A.

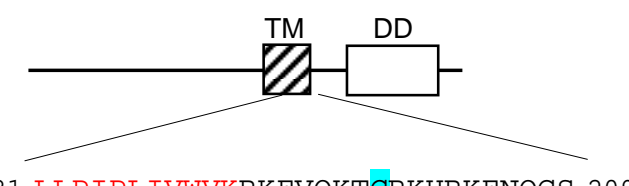

hFas 181 LLPIPLIVWVKRKEVQKTCRKHRKENQGS 209

hTNFR-1 224 LLSLLFIGLMYMYQRWKSKLYSIVCGKST 252

hDR4 252 LLLVAVLIVCCCIGSGCGGDPKCMDRVCF 280

hDR5 221 AAVVLIVAVFVCKSLLWKKVLPYLKGICS 249

B.

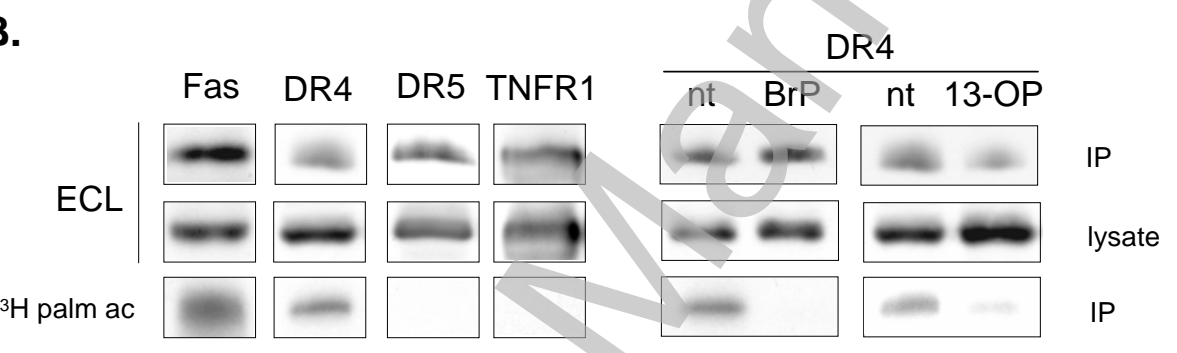

C.

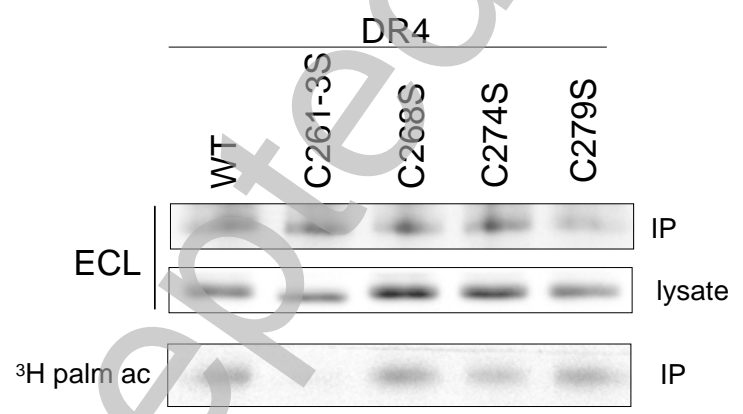

Rossin et al

Figure 1 
A.

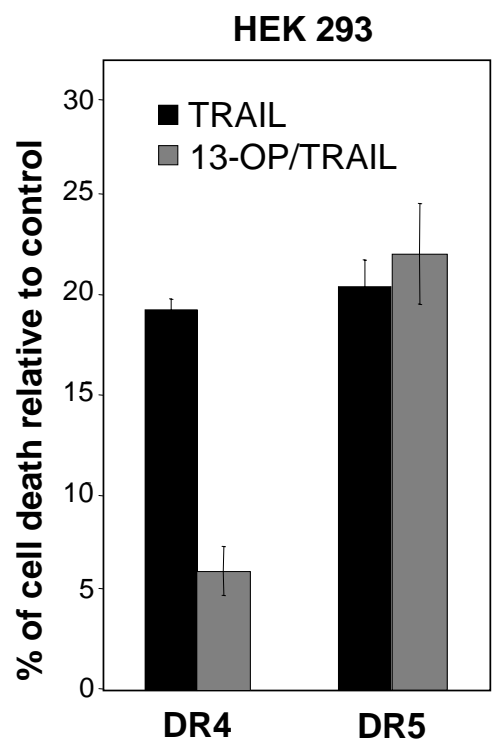

B.

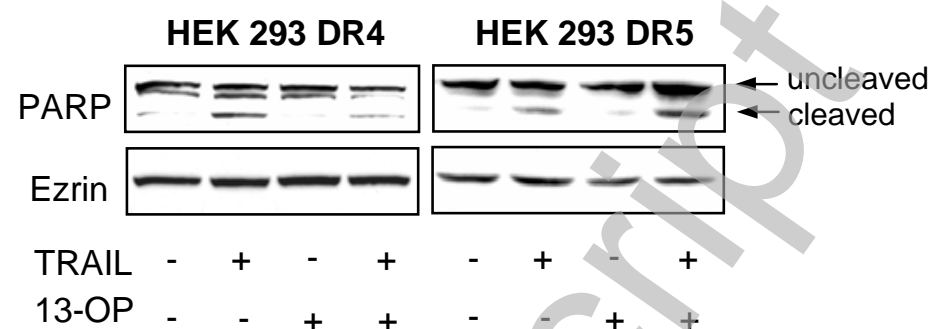

C.

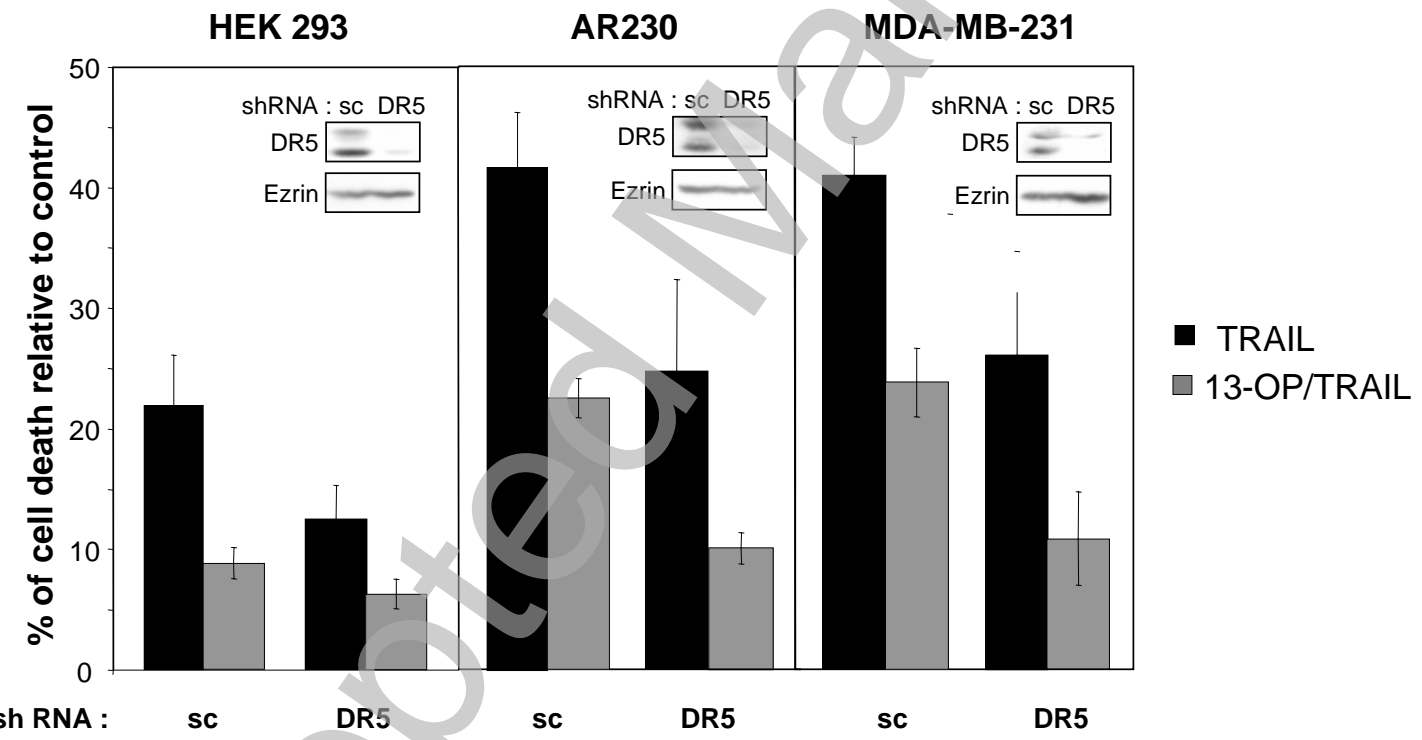

D.

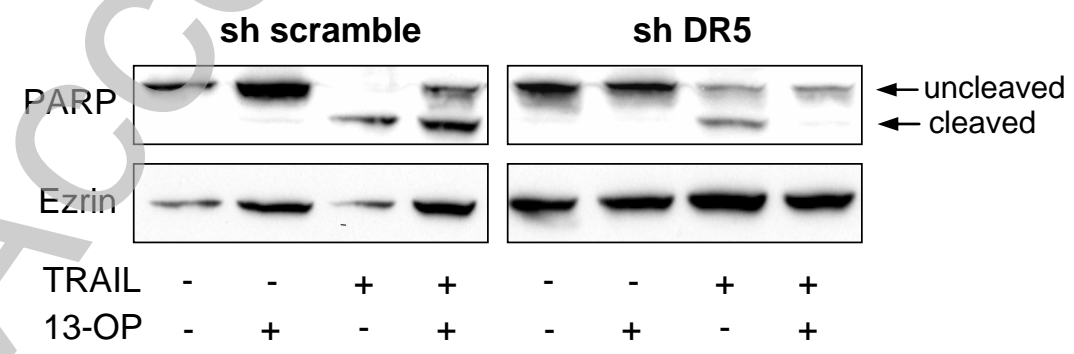

Rossin et al

Figure 2 
A.

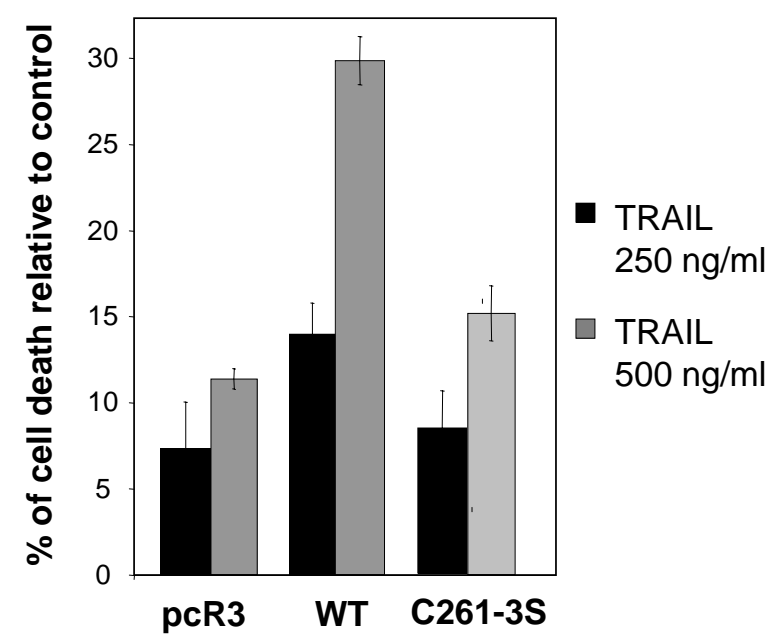

B.

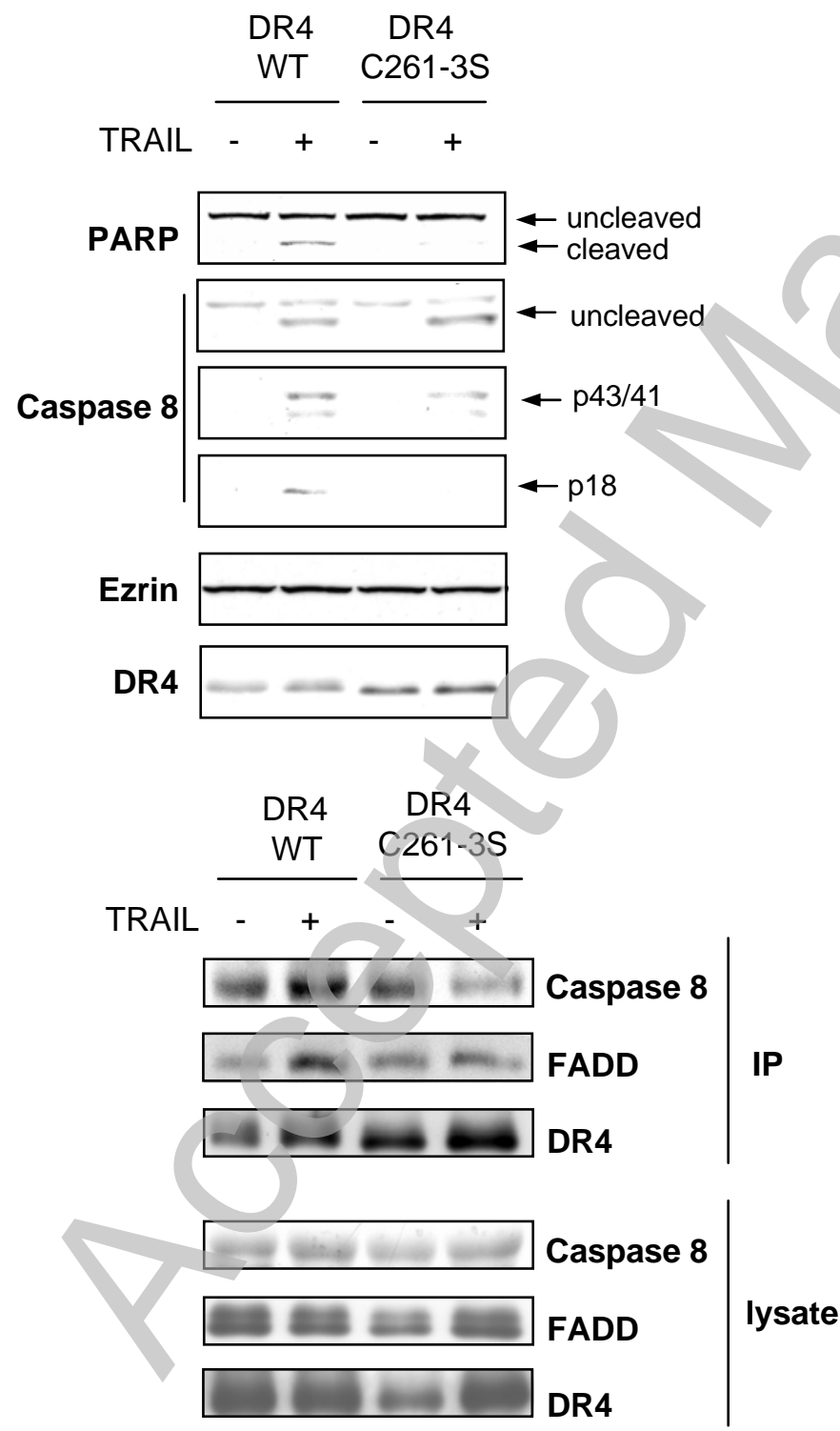

Rossin et al

Figure 3

Licenced copy. Copying is not permitted, except with prior permission and as allowed by law. (c) 2008 The Authors Journal compilation (c) 2008 Portland Press Limited 
A.

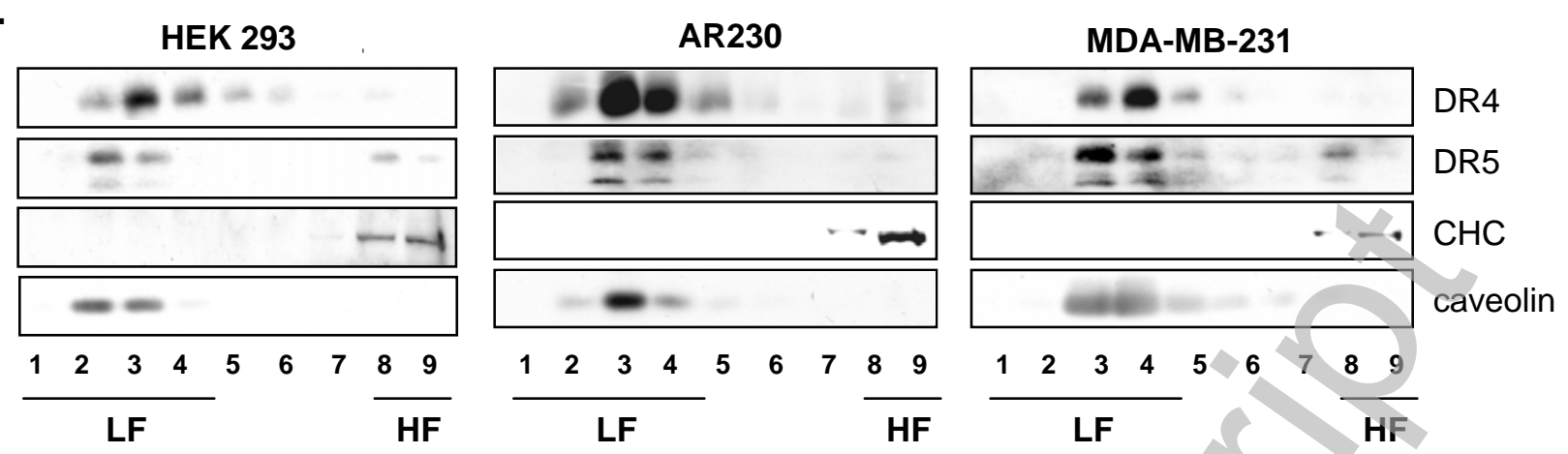

B.

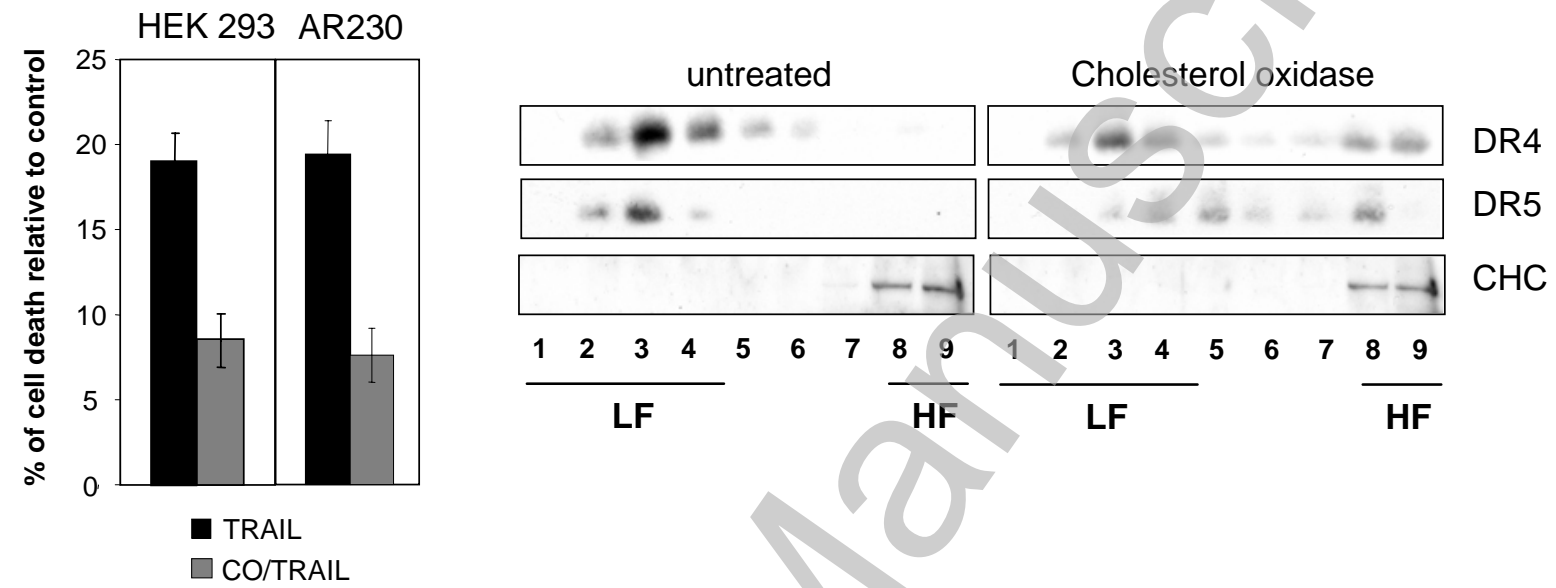

C.

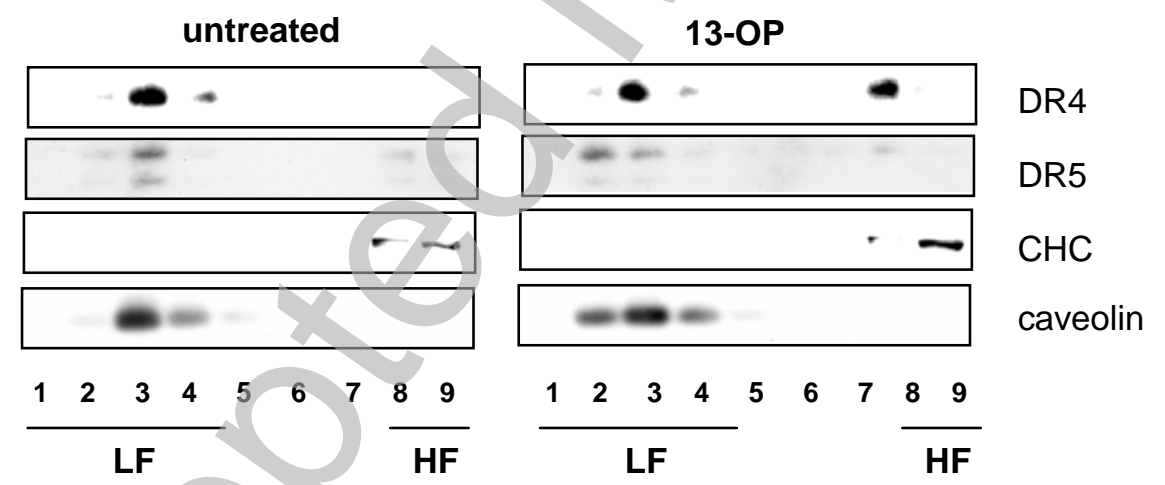

D.

DR4 C261-3S

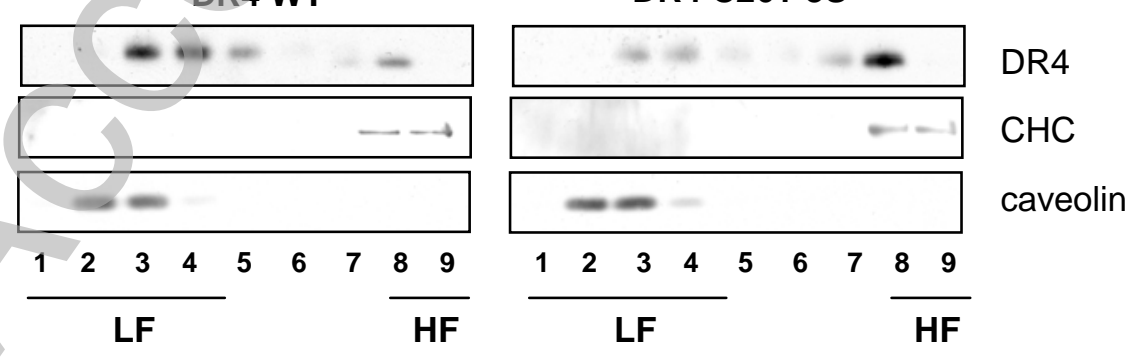

Rossin et al

Figure 4

Licenced copy. Copying is not permitted, except with prior permission and as allowed by law. (c) 2008 The Authors Journal compilation (c) 2008 Portland Press Limited 
A.

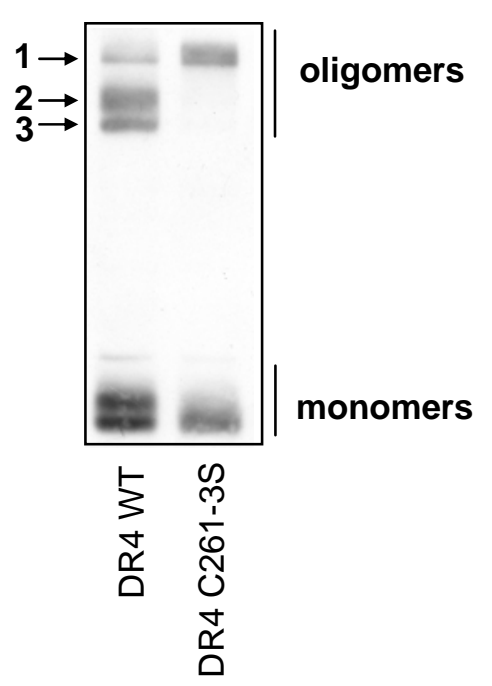

B.

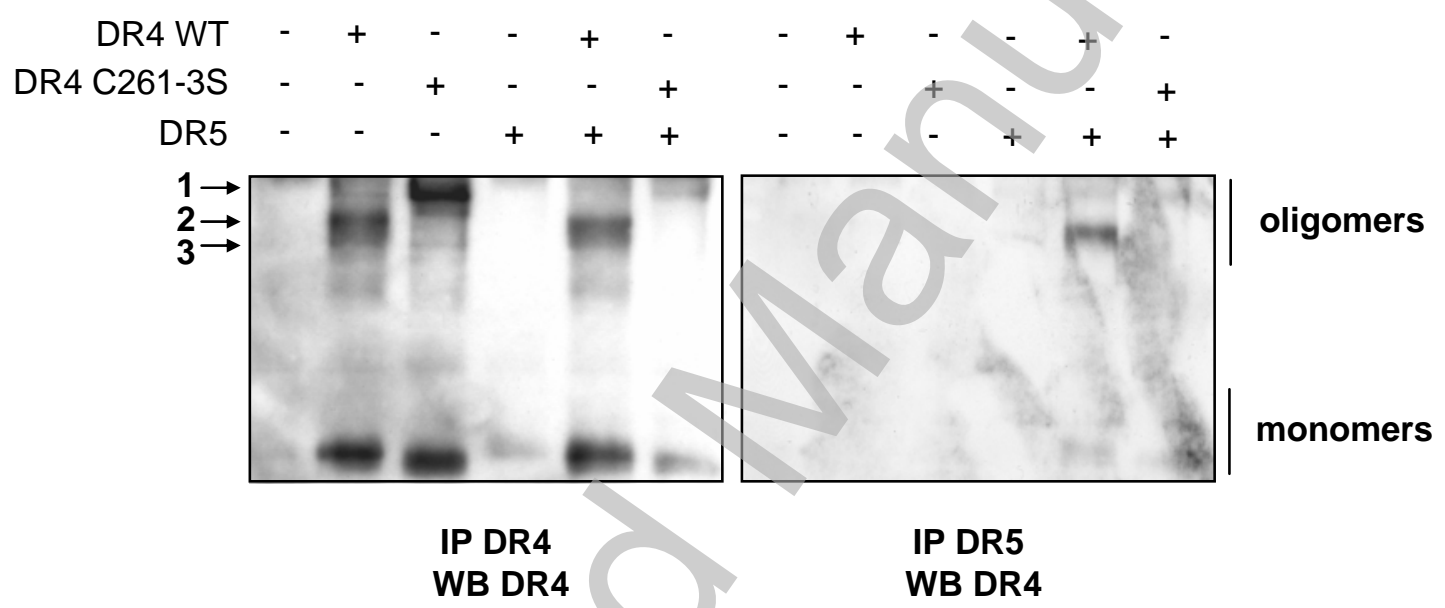

Rossin et al Figure 5 